\title{
PARACOMPACTNESS OF BOX PRODUCTS OF COMPACT SPACES ${ }^{1}$
}

\author{
BY \\ KENNETH KUNEN ${ }^{2}$
}

\begin{abstract}
We consider box products of countably many compact Hausdorff spaces. Under the continuum hypothesis, the product is paracompact iff its Lindelö degree is no more than the continuum; in particular, the product is paracompact if each space has weight continuum or less, or if each space is dispersed. Some partial results are proved under Martin's axiom.
\end{abstract}

0. Introduction. All our spaces are $T_{3}$ (regular Hausdorff). The box topology on $\Pi_{n} X_{n}$ has as a basis arbitrary products of open sets. Such a product of compact spaces is compact only in trivial cases, but it may be paracompact. By van Douwen [vD 2] there are compact $X_{n}$ of weight $\omega_{2}$ such that $\Pi_{n} X_{n}$ is not even normal. However, Rudin has shown that the continuum hypothesis (CH) implies that $\Pi_{n} X_{n}$ is paracompact if each $X_{n}$ is compact metric [R 1] or if each $X_{n}$ is a compact ordinal [R 2].

In this paper, we obtain easier proofs of some stronger positive results. Under $\mathrm{CH}$, when each $X_{n}$ is compact, $\Pi_{n} X_{n}$ is paracompact iff it is $\omega_{1-}$ Lindelöf (see 3.2). In particular if each $X_{n}$ has weight $\leqslant \omega_{1}, \Pi_{n} X_{n}$ is paracompact under $\mathrm{CH}$ (4.1); under Martin's axiom, we can modify our arguments to establish this if we assume also that the $X_{n}$ are first countable (4.4). In $\S 5$, we show that if each $X_{n}$ is a compact dispersed space, $\Pi_{n} X_{n}$ is $c$-Lindelöf and hence, under $\mathrm{CH}$, paracompact. $\$ 1$ reviews some general results on paracompact spaces, and $\$ 2$ applies them to box products. $\$ 6$ extends the results of $\$ 4$ and $\$ 5$ to products of locally compact paracompact spaces.

1. Paracompactness. We collect here some known results and easy consequences thereof.

1.1. THEOREM (MiChAEL [M 1]). The following are equivalent:

(a) $X$ is paracompact.

Received by the editors October 25, 1976.

AMS (MOS) subject classifications (1970). Primary 54B10; Secondary 54D30, 54G10.

Key words and phrases. Box product, paracompact space, dispersed space, Lindelöf degree.

'This research was supported by NSF Grant GP-27633.

${ }^{2}$ The author is grateful to the referee for a number of very helpful comments.

- American Mathematical Society 1978 
(b) Every open cover of $X$ has a $\sigma$-locally finite open refinement.

(c) Every open cover of $X$ has a $\sigma$-discrete open refinement.

We remind the reader that all our spaces are $T_{3}$, an assumption needed in the theorem. A corollary of the fact that (b) implies (a) is that Lindelöf spaces are paracompact. We shall need a generalization of this, which we state after recalling some definitions.

1.2. Definition. Let $\kappa$ be an infinite cardinal.

(1) $X$ is $\kappa$-Lindelöf iff every open cover of $X$ has a subcover of cardinality $<\kappa$.

(2) $X$ is $\kappa$-open iff the intersection of less than $\kappa$ open sets in $X$ is open.

Thus, $\omega$-Lindelöf $=$ Lindelöf, and all spaces are $\omega$-open. The fact that Lindelöf spaces are paracompact is the following lemma for $\kappa=\omega$.

1.3. Lemma. If $X$ is $\kappa$-open and $\kappa$-Lindelöf, then $X$ is paracompact.

Proof. If $\kappa>\omega$, then $X$ is zero dimensional, since it is regular and $\omega_{1}$-open. Let $\mathcal{U}$ be an open cover of $X$. Then $\mathcal{Q}$ has a refinement $\left\{V_{\alpha}\right.$ : $\alpha<\kappa\}$, where each $V_{\alpha}$ is clopen. Let

$$
W_{\alpha}=V_{\alpha}-\bigcup\left\{V_{\beta}: \beta<\alpha\right\} .
$$

Then $\left\{W_{\alpha}: \alpha<\kappa\right\}$ is a disjoint clopen refinement of $थ$.

We shall also need the following result on preservation of paracompactness under closed maps.

1.4. LeMma. Let $F$ be a closed continuous map from $X$ onto $Y$. Then

(1) If $X$ is paracompact, so is $Y$.

(2) If $Y$ is paracompact and $F^{-1}\{y\}$ is Lindelöf for each $y \in Y$, then $X$ is paracompact.

Proof. For (1), see Michael [M 2]. For (2), let $थ$ be an open cover of $X$. For each $y \in Y$, choose a countable subfamily of $\mathcal{Q},\left\{U_{n}^{y}: n \in \omega\right\}$, which covers $F^{-1}\{y\}$. Let

$$
V^{y}=\left\{z \in Y: F^{-1}\{z\} \subseteq \bigcup_{n \in \omega} U_{n}^{y}\right\} .
$$

Then $y \in V^{y}$ and $V^{y}$ is open since $F$ is closed. Let $\left\{W^{y}: y \in Y\right\}$ be a locally finite open refinement of $\left\{V^{y}: y \in Y\right\}$ which is precise (i.e., $W^{y} \subseteq V^{y}$ ). Let $\Re_{n}$ be the family $\left\{U_{n}^{y} \cap F^{-1}\left(W^{y}\right): y \in Y\right\}$. Then $\left\{\Re_{n}: n \in \omega\right\}$ forms a $\sigma$-locally finite open refinement of $\mathcal{Q}$. Thus, $X$ is paracompact by Theorem 1.1 .

We remark that (2) is well known in the case that each $F^{-1}\{y\}$ is compact, and the proof above is an easy modification of the standard proof for that case. 
Lindelöf degree has the same preservation property, with a similar, but easier proof.

1.5. LemMa. Let $F$ be a closed continuous map from $X$ onto $Y$, such that $F^{-1}\{y\}$ is Lindelöf for each $y \in Y$. Then for any infinite $\kappa, Y$ is $\kappa$-Lindelöf iff $X$ is.

2. The reduced box product. We shall define a certain reduced product, $\Pi_{n} X_{n} / \sim$. This will be easier to deal with than $\Pi_{n} X_{n}$, as it is $\omega_{1}$-open. However, for compact $X_{n}, \Pi_{n} X_{n}$ and $\Pi_{n} X_{n} / \sim$ will have similar paracompactness and Lindelöf properties. The reduced product was first defined by Knight [Kn], and was applied by Rudin [R 2] to obtain information about box products.

$\Pi_{n} X_{n} / \sim$ is defined as follows. If $p, q \in \Pi_{n} X_{n}$, we say $p$ is equivalent to $q$ $(p \sim q)$ iff $p(n)=q(n)$ for all but finitely many $n$. Let $E(p)=\{q: q \sim p\}$, and, for $A \subseteq \Pi_{n} X_{n}, E(A)=\bigcup\{E(p): p \in A\}$. We give the quotient space $\Pi_{n} X_{n} / \sim=\left\{E(p): p \in \Pi_{n} X_{n}\right\}$ the usual quotient topology and let $\sigma: \Pi_{n} X_{n}$ $\rightarrow \Pi_{n} X_{n} / \sim$ be the usual projection. So $\sigma(p)=E(p)$; we use $\sigma(p)$ when we are thinking of a point in $\Pi_{n} X_{n} / \sim$ and $E(p)$ when we are thinking of a subset of $\Pi_{n} X_{n}$.

It is easy to see that each $E(p)$ is closed. Also, the map $\sigma$ is open, so that the $\sigma(U)$ for $U$ basic in $\Pi_{n} X_{n}$ form a base for $\Pi_{n} X_{n} / \sim$. We shall rely heavily on the following

2.1. THEOREM (RUDIN). $\Pi_{n} X_{n} / \sim$ is $\omega_{1}$-open.

2.2. THEOREM. If each $X_{n}$ is compact, the map $\sigma$ is closed.

Before proving these, we remark that the interest of 2.2 is in its corollary,

2.3. COROLlaRY. If each $X_{n}$ is compact, then

(a) $\Pi_{n} X_{n}$ is paracompact iff $\Pi_{n} X_{n} / \sim$ is.

(b) For any infinite $\kappa, \Pi_{n} X_{n}$ is $\kappa$-Lindelöf iff $\Pi_{n} X_{n} / \sim$ is.

Proof. Point inverses under $\sigma$ are $\sigma$-compact, and hence Lindelöf, so 1.4 and 1.5 apply.

We turn now to the proofs of Theorems 2.1 and 2.2. 2.1 is essentially proved in [R 2], but we include a proof to show that 2.1 and 2.2 actually both follow from the same Lemma 2.4. For each $k$, let $\tau_{k}$ be the canonical projection from $\Pi_{n} X_{n}=\Pi_{n<k} X_{n} \times \Pi_{n>k} X_{n}$ onto $\Pi_{n>k} X_{n}$.

2.4. Lemma. If, for each $k, U^{k}$ is an open subset of $\Pi_{n>k} X_{n}$, then $\cap\left\{\tau_{k}^{-1}\left(U^{k}\right): k<\omega\right\}$ is open in $\Pi_{n} X_{n}$.

Proof. Fix $p \in \cap\left\{\tau_{k}^{-1}\left(U^{k}\right): k<\omega\right\}$. Then for each $k$, there is a neighborhood $V^{k}$ of $p$ contained in $\tau_{k}^{-1}\left(U^{k}\right)$, which may be taken to be of the form 


$$
V^{k}=X_{0} \times X_{1} \times \cdots \times X_{k-1} \times V_{k}^{k} \times V_{k+1}^{k} \times V_{k+2}^{k} \times \ldots,
$$

each $V_{n}^{k}(n>k)$ being open in $X_{n}$. Let $W_{n}=\cap\left\{V_{n}^{k}: k<n\right\}$, and $W=$ $\Pi_{n} W_{n}$. Then $W=\cap\left\{V^{k}: k<\omega\right\}$ is a neighborhood of $p$ contained in each $\tau_{k}^{-1}\left(U^{k}\right)$.

To deduce 2.1 from 2.4, let $\sigma_{k}$ be the map from $\Pi_{n>k} X_{n}$ onto $\Pi_{n} X_{n} / \sim$ such that $\sigma=\sigma_{k} \circ \tau_{k}$. If $V^{k}$ is open in $\Pi_{n} X_{n} / \sim$ for each $k<\omega$, then $\sigma_{k}^{-1}\left(V^{k}\right)$ is open in $\Pi_{n \geqslant k} X_{n}$, so $\sigma^{-1}\left(\cap\left\{V^{k}: k<\omega\right\}\right)$, which is equal to $\bigcap\left\{\tau_{k}^{-1}\left(\sigma_{k}^{-1}\left(V^{k}\right)\right): k<\omega\right\}$, is open in $\Pi_{n} X_{n}$, so $\cap\left\{V^{k}: k<\omega\right\}$ is open in $\Pi_{n} X_{n} / \sim$.

To deduce 2.2, note that showing $\sigma$ is closed is equivalent to showing that whenever $K$ is closed in $\Pi_{n} X_{n}$, so is $\sigma^{-1} \sigma K$. But this set is just $U\left\{\tau_{k}^{-1} \tau_{k} K\right.$ : $k<\omega\}$. Hence, if each $\tau_{k} K$ is closed in $\Pi_{n \geqslant k} X_{n}$, then by $2.4, \sigma^{-1} \sigma K$ is closed. But each $\tau_{k} K$ is closed, since projection from a compact factor is a closed map.

3. On paracompactness and the Lindelöf degree. Under $\mathrm{CH}$, our main tool for proving paracompactness is

3.1. THEOREM (CH). If each $X_{n}$ is compact, then $\Pi_{n} X_{n}$ is paracompact iff it is $\omega_{1}$-Lindelöf.

Proof. If $\Pi_{n} X_{n}$ is $\omega_{1}$-Lindelöf, then so is $\Pi_{n} X_{n} / \sim$ by 2.3. Since $\Pi_{n} X_{n} / \sim$ is also $\omega_{1}$-open by 2.1 , it is paracompact by 1.3 , so $\Pi_{n} X_{n}$ is paracompact by 2.3 .

The other direction is more difficult and is not needed for the rest of this paper. We shall in fact establish the following, without $\mathrm{CH}$.

3.2. THEOREM. If each $X_{n}$ is compact and $\Pi_{n} X_{n}$ is paracompact, then it is c-Lindelöf.

We remark that we know of no example, under any set-theoretic axioms, of a box product of compact spaces which is normal but not paracompact.

To prove 3.2, we need the following unpublished result of Arhangel'skii:

3.3. THEOREM. If $Y$ is compact and $\mathscr{F}$ is a cover of $Y$ by closed $G_{\delta}$ sets and $\mathscr{F}$ satisfies

$$
\forall H \in \mathscr{F}(|\{K \in \mathscr{F}: H \cap K \neq 0\}| \leqslant c)
$$

then $|\mathscr{F}| \leqslant c$.

Of course, if the elements of $\mathscr{F}$ are points, 3.3 is just the well-known result of [A], a simple proof of which is due to Pol [P]. Pol's proof can easily be modified to yield 3.3.

We wish to apply 3.3 with $Y$ the product of the spaces $X_{n}$ under the usual (Tychonov) topology. To do this we need

3.4. LemMa. Let $Z$ be paracompact and $\mathscr{B}$ any base for $Z$. Then every open 
cover थ for $Z$ has a refinement to a cover $\mathscr{F}$ such that

(1) Elements of $\mathscr{F}$ are of the form $\cap_{n} B_{n}$, where each $B_{n} \in \mathscr{B}$, and $B_{0} \supseteq$ $\operatorname{cl}\left(B_{1}\right) \supseteq B_{1} \supseteq \operatorname{cl}\left(B_{2}\right) \supseteq \ldots$

(2) $\mathscr{F}$ satisfies $(*)$.

We apply 3.4 with $Z$ the box product $\Pi_{n} X_{n}$ and $\mathscr{B}$ the usual base. Then elements of $\mathscr{F}$ will be closed $G_{\delta}$ sets in $Y$, so $|\mathscr{F}| \leqslant c$ by 3.3. Thus, if $Z$ is paracompact, it is $c$-Lindelöf.

To prove 3.4, we apply paracompactness $\omega$ times to produce a sequence of covers $\mathfrak{T}_{n}(n \in \omega)$ of $Z$ with $\mathfrak{V}_{0}=\mathcal{Q}$ and

(a) Each $\widetilde{V}_{n+1}$ is a locally finite open refinement of $\mathfrak{V}_{n}$.

(b) If $V_{n+1} \in \mathfrak{V}_{n+1}, V_{n+1}$ intersects only finitely many members of $\mathfrak{V}_{n}$.

(c) If $V_{n+1} \in \mathfrak{V}_{n+1}$, there is a $B \in \mathscr{B}$ and $V_{n} \in \mathfrak{V}_{n}$ with $V_{n+1} \subseteq B \subseteq \operatorname{cl}(B)$ $\subseteq V_{n}$.

Let $\mathscr{F}$ be the set of all sets of the form $\bigcap_{n} V_{n}=\bigcap_{n} B_{n}$, where each $V_{n} \in \mathscr{V}_{n}, B_{n} \in \mathscr{B}$, and $V_{0} \supseteq \operatorname{cl}\left(B_{0}\right) \supseteq B_{0} \supseteq V_{1} \supseteq \ldots \mathscr{F}$ satisfies (*) by conditon (b). To see that $\mathscr{F}$ is a cover, fix $p \in X$. Let $T$ be the tree of all finite sequences $\left\langle V_{1} \ldots V_{k}\right\rangle$ such that $p \in V_{k}$, each $V_{n} \in \mathfrak{V}_{n}$, and for some $B_{1} \ldots B_{k-1} \in \mathscr{B}, V_{1} \supseteq \operatorname{cl}\left(B_{1}\right) \supseteq B_{1} \supseteq V_{2} \supseteq \cdots \supseteq B_{k-1} \supseteq V_{k}$. Each level of $T$ is finite, since the covers are point finite, but $T$ is infinite, so by König's lemma, there is a path through $T$. The intersection of this path is a set in $\mathscr{F}$ containing $p$.

4. Trivial applications of $\$ \$ 1-3$. Theorem 3.1 yields immediately:

4.1. THEOREM (CH). If each $X_{n}$ is compact and of weight $<\omega_{1}$, then $\Pi_{n} X_{n}$ is paracompact.

We would call an application of $\$ \S 1-3$ nontrivial if the Lindelöf degree of $\Pi_{n} X_{n} / \sim$ is established by some bound other than its weight. For example, in $\$ 5$ we show that if each $X_{n}$ is a compact dispersed space, $\Pi_{n} X_{n} / \sim$ is $c$-Lindelöf.

We look now at the situation under Martin's axiom (MA). See Jech [J] for a complete discussion of MA, including a proof of its consistency with $\neg \mathrm{CH}$. We need here only the well-known

4.2. Lemma. Assume MA. If $\kappa<c$ and $f_{\alpha}(\alpha<\kappa)$ are functions from $\omega$ into $\omega$, then there is a $g: \omega \rightarrow \omega$ such that for each $\alpha, g(n)>f_{\alpha}(n)$ for all but finitely many $n$.

This gives us, in analogy with Theorem 2.1,

4.3. THEOREM. If MA and each $X_{n}$ is first countable, then $\Pi_{n} X_{n} / \sim$ is c-open.

Proof. Fix $\sigma(p) \in \Pi_{n} X_{n} / \sim$ and let $U^{\alpha}(\alpha<\kappa)$ be neighborhoods of $\sigma(p)$, where $\kappa<c$. We show that $\cap\left\{U_{\alpha}: \alpha<\kappa\right\}$ is a neighborhood of $\sigma(p)$. For 
each $n$, let $\left\{V_{n}^{k}: k<\omega\right\}$ be a base at $p(n)$ in $X_{n}$. For each $\alpha$, let $f_{\alpha}: \omega \rightarrow \omega$ be such that

$$
\sigma\left(\prod_{n} V_{n}^{f_{n}(n)}\right) \subseteq U_{\alpha}
$$

Then if $g$ is as in 4.2, $\sigma\left(\Pi_{n} V_{n}^{g(n)}\right)$ is an open neighborhood of $\sigma(p)$ contained in $\cap\left\{U_{\alpha}: \alpha<\kappa\right\}$.

Then, analogously to 4.1 , we have the following, whose proof is obtained essentially by replacing $\omega_{1}$ by $c$ in the proof of the easy direction of 3.1.

4.4. THEOREM (MA). If each $X_{n}$ is compact and first countable, then $\Pi_{n} X_{n}$ is paracompact.

Proof. By Arhangel'skii [A], each $X_{n}$ has cardinality $<c$, and thus weight $\leqslant c$, so $\Pi_{n} X_{n} / \sim$ has weight $\leqslant c$, so by $4.3, \Pi_{n} X_{n} / \sim$, and hence also $\Pi_{n} X_{n}$ is paracompact.

We do not know whether $\mathrm{CH}$ is required for Theorem 4.1, or MA for 4.4. van Douwen [vD 3] has shown that in 4.4, if each $X_{n}$ is compact metric, then MA may be weakened to the existence of a $\kappa$-scale in $\omega^{\omega}$ for some $\kappa$. The proof shows that in this case, $\Pi_{n} X_{n} / \sim$ is $\kappa$-metrizable.

Under $7 \mathrm{CH}$, there is no criterion like Theorem 3.1 for paracompactness in terms of Lindelöf degree. By van Douwen [VD 2], there are always compact $X_{n}$ of weight $\omega_{2}$ such that $\Pi_{n} X_{n}$ is not even normal; under $7 \mathrm{CH}$, this box product would have weight $c$ and hence Lindelöf degree $c$ (since no nontrivial box product has Lindelöf degree $<c$ ). But the box product of 2-point spaces has the same Lindelöf degree $c$ and is paracompact (in fact discrete).

5. Products of compact dispersed spaces. Rudin [R 2] shows that under $\mathbf{C H}$, if each $X_{n}$ is a compact ordinal (i.e., a successor ordinal with the order topology), then $\Pi_{n} X_{n}$ is paracompact. An easier proof of this may be obtained by first establishing without any set-theoretic assumptions, that $\Pi_{n} X_{n}$ is $c$-Lindelöf, and then quoting 3.1. Following a suggestion of Scott Williams, we can also generalize this to dispersed spaces:

5.1. THEOREM. If each $X_{n}$ is a compact dispersed space, then $\Pi_{n} X_{n}$ is c-Lindelöf.

Here, $X$ is dispersed iff each subspace $Y$ contains an isolated (in $Y$ ) point. Ordinals are dispersed, since the first point in any subspace of an ordinal is isolated.

For any $X$, one can form the Cantor-Bendixon sequence $X^{(\alpha)}$, where $X^{(0)}=X, X^{(\alpha+1)}=\left\{x \in X^{(\alpha)}: x\right.$ is not isolated in $\left.X^{(\alpha)}\right\}$, and $X^{(\gamma)}=$ $\cap\left\{X^{(\alpha)}: \alpha<\gamma\right\}$ for $\gamma$ a limit. Then each $X^{(\alpha)}$ is closed in $X$, the $X^{(\alpha)}$ are nonincreasing, and, if $Y \subseteq X, Y^{(\alpha)} \subseteq X^{(\alpha)}$ for all $\alpha$. $X$ is dispersed iff $X^{(\alpha)}=0$ for some $\alpha$. If $X$ is compact dispersed, then the first $\alpha$ such that 
$X^{(\alpha)}=0$ is a successor ordinal, $\beta+1$, and $X^{(\beta)}$ is finite; we call $\beta$ the rank of $X$.

To prove Theorem 5.1, let $थ$ be an open cover of $\Pi_{n} X_{n}$. Following [R 2], we use a tree to index our attempts to refine $\mathcal{Q}$, but we look for a closed rather than an open refinement.

Let $T=c^{<\omega_{1}}=\bigcup\left\{c^{\xi}: \xi<\omega_{1}\right\}$. Elements $s \in T$ are viewed as $c$-ary sequences of countable ordinal length, so $T$ is the complete $c$-ary tree of height $\omega_{1}$. We write $\operatorname{lh}(s)$ for the length (domain) of $s$, and, if $\eta<\operatorname{lh}(s), s \uparrow \eta$ is the sequence of length $\eta$ formed by restricting $s$ to $\eta$. If $s \in T$ and $\mu \in c$, then $s \mu$ is the sequence of length $\operatorname{lh}(s)+1$ which begins with $s$ and ends with the element $\mu .0$ is the empty sequence, of length 0 .

$K \triangleleft \mathcal{Q}$ means $\exists U \in \mathcal{Q}(K \subseteq U)$. We shall define, by induction on $\operatorname{lh}(s)$, $K(s)=\Pi_{n} K_{n}(s)$, a closed box in $\Pi_{n} X_{n}$. We shall then check that $\forall p \in \Pi_{n} X_{n}$ $\exists s \in T(p \in K(s) \triangleleft \mathcal{Q})$, which, since $T$ has cardinality $c$, will imply that $\mathscr{U}$ has a subcover of cardinality $c$.

$K(s)$ will be defined so that $K(0)=\Pi_{n} X_{n}$, and, for each 's $\in T$,

(1) $K(s)=\bigcup\{K(s \mu): \mu<c\}$.

(2) If $\operatorname{lh}(s)$ is a limit, $K(s)=\bigcap\{K(s \mid \xi): \xi<\operatorname{lh}(s)\}$.

(3) For each $\mu<c$, either $K(s \mu) \triangleleft$ थ or $\exists n\left[\operatorname{rank}\left(K_{n}(s \mu)\right)<\operatorname{rank}\left(K_{n}(s)\right)\right]$.

By (1) and (2), if $p \in \Pi_{n} X_{n}$, there is an $f \in c^{\omega_{1}}$ with $p \in K(f \uparrow \xi)$ for all $\xi<\omega_{1}$. Then, for each $n$, the ranks of the $K_{n}(f \uparrow \xi)$ are nonincreasing and hence eventually constant. If they are constant past $\xi$ for each $n$, then (3) insures that $K(f \uparrow \xi) \triangleleft$ थ. Hence, we shall be done if (1)-(3) can be accomplished.

To define $K(s)$, we proceed by induction, taking intersections at limits, so that (2) holds by definition. For a successor stage, fix $s$. Let $\beta_{n}=\operatorname{rank}\left(K_{n}(s)\right)$ and $Y_{n}=\left(K_{n}(s)\right)^{\left(\beta_{n}\right)}$. Then $Y_{n}$ is finite. Let $\mathcal{V}$ be a family of open boxes of cardinality $c$ such that $\mathcal{V}$ covers $\Pi_{n} Y_{n}$ and $\operatorname{cl}(V) \triangleleft \mathcal{Q}$ for all $V \in \mathcal{V}$. This is possible since $\left|\Pi_{n} Y_{n}\right|<c$. Let $K(s \mu)$, for $\mu<c$, enumerate all the $c$ boxes $K=\Pi_{n} K_{n}$ such that either

(a) $K=\operatorname{cl}(V) \cap K(s)$ for some $V \in \mathfrak{V}$ or

(b) for some $n$,

(i) $K_{n}=K_{n}(s)-\left(V_{n}^{1} \cup \cdots \cup V_{n}^{j}\right)$ for some $V^{1} \ldots V^{j} \in \mathfrak{V}$ with $Y_{n} \subseteq$ $V_{n}^{1} \cup \ldots \cup V_{n}^{j}$ and

(ii) $K_{m}=K_{m}(s)$ for all $m \neq n$.

Then (3) holds, since (b)(i) implies that $\left(K_{n}\right)^{\left(\beta_{n}\right)}=0$, whence $\operatorname{rank}\left(K_{n}\right)<$ $\beta_{n}$. To check (1), fix $p \in K(s)$. If $p$ fails to be in any of the $K(s \mu)$ of type (b) then, since $Y_{n}$ is finite, for each $n$ there must be a $q_{n} \in Y_{n}$ with $\forall V \in \mathcal{V}$ $\left(q_{n} \in V_{n} \rightarrow p_{n} \in V_{n}\right)$. Let $q=\left\langle q_{0} q_{1} \ldots\right\rangle \in \Pi_{n} Y_{n}$. Then $\forall V \in \mathcal{V}(q \in V \rightarrow$ $p \in V)$, so, taking $V$ containing $q, p$ is in $\operatorname{cl}(V) \cap K(s)$, which is one of the $K(s \mu)$ of the type (a). 
Thus, the $K(s)$ may indeed be defined to satisfy (1)-(3), which completes the proof of 5.1.

5.1 can be improved by using a modified Cantor-Bendixon analysis. Let $w(Z)$ denote the weight of the space $Z$. For any $X$, let $X^{\prime}=X-\cup\{N: N$ is open and $w(N)<c\}$; let $X^{[0]}=X, X^{[\alpha+1]}=\left(X^{[\alpha]}\right)^{\prime}$, and take intersections at limits as before. Call $X$ almost dispersed iff $X^{[\alpha]}$ is empty for some $\alpha$ (equivalently, iff every subspace contains a neighborhood of weight $\leqslant c$ ). The class of almost dispersed spaces contains all dispersed spaces and all spaces of weight $\leqslant c$, and is closed under countable box products.

5.2. THEOREM. If each $X_{n}$ is compact and almost dispersed, then $\Pi_{n} X_{n}$ is c-Lindelöf.

The proof is as for 5.1, with the appropriate modification in the notion of rank. In the justification for the existence of $\mathcal{T}$, one uses that $\Pi_{n} Y_{n}$ has weight $<c$, rather than cardinality $<c$. In the argument establishing (1), use the fact that the $Y_{n}$ are compact, rather than finite.

Then, by 3.1, we have

5.3. THEOREM (CH). If each $X_{n}$ is compact and almost dispersed, then $\Pi_{n} X_{n}$ is paracompact.

6. Products of locally compact spaces. Compactness can be replaced by local compactness plus paracompactness in the positive results of $\$ 4$ and $\S 5$ by

6.1. THEOREM. Assume that each $X_{n}$ is paracompact and has an open cover $\mathcal{Q}_{n}$ such that whenever $U_{n} \in \mathcal{U}_{n}(n \in \omega), \Pi_{n} \operatorname{cl}\left(U_{n}\right)$ is paracompact. Then $\Pi_{n} X_{n}$ is paracompact.

Thus, e.g., by $4.1,4.4$ and 5.3,

6.2. Corollary. Assume each $X_{n}$ is a paracompact and locally compact.

(a) (CH) If each $X_{n}$ is locally of weight $\leqslant \omega_{1}$, then $\Pi_{n} X_{n}$ is paracompact.

(b) (MA) If each $X_{n}$ is first countable, then $\Pi_{n} X_{n}$ is paracompact.

(c) $(\mathrm{CH})$ If each $X_{n}$ is dispersed, then $\Pi_{n} X_{n}$ is paracompact.

The assumption of paracompactness of the $X_{n}$ cannot be dropped from these results, since each $X_{n}$ is homeomorphic to a closed subspace of $\Pi_{n} X_{n}$. Also, under $\mathrm{CH}, \omega_{1} \times(\omega+1)^{\omega}$ is not even normal (see [Ku]). A special case of $6.2(\mathrm{~b})$ is that under MA, the box product of locally compact metric spaces is paracompact. Under CH, this is essentially due to Rudin [R 1], as was pointed out by van Douwen [VD 1]. [VD 1] also shows that the box product of non-locally-compact separable metric spaces need not be normal.

To prove 6.1, note first that we can, by criterion (c) of Michael's Theorem 1.1 , assume that each $\mathscr{Q}_{n}$ is countable; if not, we pass to $\sigma$-discrete refinements of the $\mathscr{Q}_{n}$ and note that a discrete union of paracompact spaces is 
paracompact. Again using paracompactness of the $X_{n}$, we may assume that each $\mathscr{U}_{n}$ is locally finite.

Say $\mathscr{Q}_{n}=\left\{U_{n}^{k}: k<\omega\right\}$. By normality, we can find closed $F_{n}^{k} \subseteq U_{n}^{k}$ such that $\cup_{k} F_{n}^{k}=X_{n}$. Then, there are open $V_{n}^{k, i}(i<\omega)$ such that

$$
U_{n}^{k} \supseteq \operatorname{cl} \dot{V}_{n}^{k, 0} \supseteq V_{n}^{k, 0} \subseteq \operatorname{cl} V_{n}^{k, 1} \supseteq \cdots \supseteq F_{n}^{k} .
$$

Whenever $f \in \omega^{\omega}$, let

$$
A_{f}=\bigcap_{i} E\left(\prod_{n} V_{n}^{f(n), i}\right)=\bigcap_{i} E\left(\prod_{n} \mathrm{cl} V_{n}^{f(n), i}\right) .
$$

Then the $A_{f}$ cover $\Pi_{n} X_{n}$. Each $A_{f}$ is closed, as it is the intersection of closed sets, and it is open, as it is the intersection of $\omega$ open sets and $\Pi_{n} X_{n} / \sim$ is $\omega_{1}$-open.

Furthermore, each $A_{f}$ is paracompact in its relative topology. To see this, note that $A_{f}$ is covered by the interiors of the $\Pi_{n} \mathrm{cl}\left(U_{n}^{g(n)}\right)$, where $g$ ranges over the countably many elements of $\omega^{\omega}$ which are eventually equal to $f$. Also, each $\Pi_{n} \mathrm{cl}\left(U_{n}^{g(n)}\right)$ is paracompact by assumption. Paracompactness of $A_{f}$ now follows immediately from the following easy general fact:

6.3. Lemma. If $Y=\cup_{j} \operatorname{int}\left(K_{j}\right)$, where each $K_{j}$ is closed and paracompact, then $Y$ is paracompact.

Proof. If $\mathcal{Q}$ is an open cover of $Y$, for each $j$ let $\mathcal{V}_{j}$ be a family of open (in $K_{j}$ ) subsets of $K_{j}$ which refines $Q$, covers $K_{j}$, and is locally finite in $K_{j}$. Let $\mathscr{W}_{j}=\left\{V \cap \operatorname{int}\left(K_{j}\right): V \in \mathcal{V}_{j}\right\} . \mathcal{W}_{j}$ is a locally finite and open (in $Y$ ) refinement of $\mathcal{Q}$ which covers int $\left(K_{j}\right)$ so the $\mho_{j}$ for $j<\omega$ form a $\sigma$-locally finite open refinement of $U$ which cover $Y$. Hence by Michael's Theorem 1.1, $Y$ is paracompact.

Finally, the family of $A_{f}$ for $f \in \omega^{\omega}$ is closure-preserving; equivalently, since the $A_{f}$ are closed, each $p$ has a neighborhood $W$ which is disjoint from all the $A_{f}$ not containing $p$. To see this, let $W=\Pi_{n} W_{n}$, where

$$
W_{n}=X_{n}-\bigcup\left\{\mathrm{cl} V_{n}^{k, i}: i, k \in \omega \text { and } p(n) \notin \mathrm{cl} V_{n}^{k, i}\right\} .
$$

$W_{n}$ is open since $\mathcal{Q}_{n}$ is locally finite. If $p \notin A_{f}$, then for some $i, p \notin$ $E\left(\Pi_{n} \mathrm{cl} V_{n}^{f(n), i}\right)$. Thus, $W_{n} \cap \operatorname{cl} V_{n}^{f(n), i}=0$ for infinitely many $n$, so $W \cap A_{f}=$ 0 .

Thus, if we let $f_{\alpha}(\alpha<c)$ enumerate $\omega^{\omega}$ and let

$$
B_{\alpha}=A_{f_{\alpha}}-\bigcup\left\{A_{f_{\beta}}: \beta<\alpha\right\},
$$

then $\Pi_{n} X_{n}$ is expressed as $\cup_{\alpha} B_{\alpha}$, a disjoint union of clopen paracompact subspaces, and is hence paracompact.

\section{REFERENCES}

[A] A. V. Arhangel'skii, The power of bicompacta with first axiom of countability, Dokl. Akad. Nauk SSSR 187 (1969), 967-968 = Soviet Math. Dokl. 10 (1969), 951-955. 
[VD 1] E. K. van Douwen, The box product of countably many metrizable spaces need not be normal, Fund. Math. 88 (1975), 127-132.

[vD 2] __, Another non-normal box product, General Topology and Appl. 7 (1977), 71-76.

[VD 3] ___ Separation and covering properties of box products and products (to appear).

[J] T. Jech, Lectures in set theory, Lecture Notes in Math., vol. 217, Springer-Verlag, Berlin and New York, 1971.

[Kn] C. J. Knight, The box topology, Quart. J. Math. Oxford Ser. (2) 15 (1964), 41-54.

[Ku] K. Kunen, Some comments on box products, Colloq. Math. Soc. János Bolyai, Infinite and finite sets, Vol. 10, Keszthely, 1973, pp. 1011-1016.

[M 1] E. Michael, A note on paracompact spaces, Proc. Amer. Math. Soc. 4 (1953), 831-838.

[M 2] __, Another note on paracompact spaces, Proc. Amer. Math. Soc. 8 (1957), 822-828.

[P] R. Pol, Short proofs of two theorems on cardinality of topological spaces, Bull. Acad. Polon Sci. 22 (1974), 1245-1249.

[R 1] M. E. Rudin, The box product of countably many compact metric spaces, General Topology and Appl. 2 (1972), 293-298.

[R 2] __, Countable box products of ordinals, Trans. Amer. Math. Soc. 192 (1974), 121-128.

Department of Mathematics, University of Wisconsin, Madison, Wisconsin 53706 